\title{
Concluding Remarks, or Pilgrim's Progress: The Road Ahead
}

\author{
Wesley A. Traub ${ }^{1,2} \dagger$ \\ ${ }^{1}$ Jet Propulsion Laboratory, M/S 301-451, 4800 Oak Grove Dr., Pasadena, CA 91109, USA \\ email: wtraub@jpl.nasa.gov \\ ${ }^{2}$ Harvard-Smithsonian Center for Astrophysics, 60 Garden St., Cambridge, MA 02138, USA
}

\begin{abstract}
This has been a wonderful conference, in a beautiful setting, with many of the world's planet-seekers on hand to present their ideas, at a time when we stand technically almost ready to embark on designing space missions to search for Earth-like planets and signs of life on them. In these remarks, I wish to simply list the 'big questions' that face us today, and offer some personal comments on how we might address these questions.

For a bit of inspiration from the past, I will close with a parallel that might be drawn between the journey that we all wish to embark upon, to search for extrasolar planets and to characterize them, and the one described by John Bunyan in his 1678 allegory, Pilgrim's Progress.

A remarkable aspect of this conference has been the agreement on our common goal, to detect and characterize extrasolar planets. While it is true that various of us suscribe to different theories of how planetary systems are formed, or how to best build the telescopes that can investigate these planetary systems, nevertheless we do all believe that the next great frontier in astrophysics is the study of extrasolar planets, and in particular Earth-like planets.

The topic of this conference can be phrased in terms of the following 'big questions'. Are there Earth-like planets around nearby stars? Are these planets habitable? Do they show signs of life?

These questions generate many sub-questions that we need to answer. In the following I list the questions as I see them today. I will phrase them in terms that can be easily understood by most of the public. Doubtless you will have other questions, but this is a start.
\end{abstract}

Keywords. stars:planetary systems, techniques: high angular resolution

\section{What does a 'normal' planetary system look like?}

Until 1995 most astronomers thought that other planetary systems probably looked like the Solar System. Since then radial-velocity (RV) data have shown that giant planets orbiting very close to their parent stars are clearly the (observationally-biased) norm. Models continue to predict that planetary systems are probably formed more along traditional lines (i.e., roughly similar to the Solar System), and that migration is required to produce the RV-observed systems. Improved RV data will permit us to see more planets in existing systems, if they are there, but we are not likely to reach the terrestrial planet range with this technique. We need Kepler and SIM to tell us more, but these too are slightly biased toward large inner planets. Ultimately we need images from TPF-C and TPF-I to tell us what the systems look like, although each of these has its own biases as well. Regarding zodiacal dust, recall that we are at present quite uncertain about whether the median planetary system contains a lot of dust, or a little. By itself the level of dust will tell us about processes in the system, but in addition we are hoping that a relatively low level of dust is present because this will keep the background noise low, and make it easier for TPF-C or TPF-I to find and characterize the planet.

$\dagger$ Present location: Jet Propulsion Laboratory 


\section{What are the characteristics of its terrestrials, giants, and disk?}

In addition to knowing that an object simply exists, we also need to know the physical and chemical characteristics of the terrestrial planets, giant planets, and zodiacal dust (and gas) in a planetary system. For this we first need to obtain colors and spectroscopy, following the traditional astronomical path of measurement priorities. Planets are likely to be even more diverse than stars, so it is not obvious how color, for example, tells us about a planet, but by the twin routes of modeling and observation in the Solar System, we can expect to learn how to characterize a planet using color. Of course, color is discussed before spectroscopy because the targets are very faint, and the first information we can expect to have is existence, followed by color, and after much more integration time, spectroscopy. The advantage of spectroscopy is that it directly relates to atomic and molecular species (as color does not). We can add to this list the properties of surfaces, both solid and cloudy, as something that spectroscopy will tell us about. Finally recall that the color of the thermal-infrared continuum will in principle tell us about the effective temperature, however complications of clouds and spectral bands will make this interpretation problematic unless we also understand something about the spectrum.

\section{What is the frequency of occurrence of 'normal' systems?}

Asking about the probability of finding a 'normal' stellar system is a broader version of the question, what is the probability of finding an Earth-like planet in the habitable zone? From Kepler and SIM we will get distribution functions, biased toward the several-Earth size and mass range, but with minimum detectable values sufficiently close to the oneEarth level that we will be able to extrapolate to that level with reasonable confidence. However we will need to image systems with TPF-C and TPF-I to know with certainty if the planets in the one-Earth range are present, and if present, what types of planets they are. Thus even though we expect that Kepler and SIM will tell us the distribution function of planets in the mass range down to about an Earth, we will also need to spectroscopically characterize an Earth-size object to find out if it is Venus-like, Marslike, or truly Earth-like.

\section{Do Earth-like planets exist nearby?}

Kepler will tell us about the likely abundance of Earth-size planets around its target stars, but these stars are several hundred parsecs distant, which is too far to be able to characterize them using either TPF-C or TPF-I. Therefore we will also need SIM to make a close examination of nearby planets, where it will be most sensitive, to tell us if Earth-mass planets are in the Solar neighborhood (roughly less than $30 \mathrm{pc}$ ). TPF-C and TPF-I can also search this same set of nearby stars, but at the cost of spending time searching instead of characterizing, and at the cost of not having dynamical masses for the objects that they will see in common with SIM.

\section{Does life exist on these planets?}

The search for life in the universe, or even around nearby stars, has progressed over the past 10 years from being a topic that was essentially the exclusive domain of supermarket tabloids, to a topic that professional scientists can now discuss in public without fear of being laughed at by their colleagues. My personal view is that the goal of astrophysics is to explain the evolution of the entire Universe, starting with the Big Bang, followed 
by galaxy formation, star formation, planet formation, and the development of life on a planet. To defend the last point, recall that major issues in planet formation include the following: the initial presence or delivery of water to a planet, the degree of bombardment suffered by a planet, the influence of radiation, the possible delivery of complex molecules, the origin and protective role of planetary magnetic fields, the influence of massive planets in the same system, etc. All these elements, and more, can have a major influence on whether life can start and flourish on a planet, and all of these are in the proper domain of astrophysics.

We have within our grasp the technical means to detect the likely presence of life on an extrasolar planet. I emphasize 'likely' because we do not have a handy 'life molecule' whose signature will tell us unequivocally that it was produced by living organisms. (However a laser beacon from a planet would do quite nicely!) What we do have is spectral signatures of molecules whose presence strongly indicates the presence of life. (a) The strongest of these is nitrous oxide, which can only be produced (in quantity) by life; unfortunately it is present only in a blended region of the infrared spectrum overlapped by water and methane, and also carbon dioxide. However missions beyond TPF-I, with greater collecting area and resolving power, could unambiguously identify this species. (b) The presence of a large amount of molecular oxygen is a good indicator of life, because only life can produce this species in abundance. Photochemistry can generate small amounts, but other chemical pathways consume it before long. Only the sustained production by life can overcome the loss mechanisms, and produce a high abundance. Ozone, produced photochemically from oxygen, is a good secondary indicator of oxygen.

(c) The simultaneous presence of a reduced species with an oxidizing species is a good indicator of a system that is not in local thermodynamic equilibrium, and in particular, life. Thus, for example, if we can see both methane and oxygen on the same planet, we will have a good indication of life. (d) Habitability is a precondition for life, and is indicated by the combination of surface temperature and water vapor, together pointing to liquid water on the surface. (e) The 'red edge' of vegetation on land indicates the presence of photosynthesis in the leaves of land plants on Earth. It is conceivable that a different process operates on another planet, for example a 3-photon one in place of the Earth's 2-photon process, which might move the red edge farther to the red. (f) Other indicators of environments conducive to life might include the presence of a substantial, cloud-free atmosphere (by Rayleigh scattering), active weather systems (from random variations of cloud reflectance or emission), and rotation period (from periodic variations of reflectance or emission).

\section{What information can we learn about a planet?}

All of the above indicators of life on a planet can be detected spectroscopically in the visible, infrared, or both. In addition we can get mass from SIM, and size from the occasional instance of a transit. We get effective temperature from the thermal infrared, and effective area as well. From mass and area we get density, hence the type of planet (ice, gas, rock). From visible brightness and area we get albedo, hence the type of reflecting material (in broad terms, gas, cloud, rock, or a combination). From mass and radius we get surface gravity. Combined with temperature we get the lapse rate. Rayleigh scattering gives the total air mass above a reflecting surface. Spectroscopy tells us the degree of greenhouse warming, and the altitude of the effective temperature, from which we can extrapolate (using the lapse rate) to get surface temperature. The temperature and spectrum will tell us if liquid water is present on the surface, and therefore if any initially large amount of carbon dioxide might have been weathered into rock. Mass, gravity, and 
density will suggest if plate tectonics might be active, and therefore if the sequestered carbon dioxide may have been pushed further below the surface. The variation of reflectivity will indicate possible land-ocean partitioning, and thus the fraction of land and water.

\section{How do we prepare ourselves, scientifically, to learn about expected, and unexpected, characteristics of extrasolar planets?}

From a combination of observation and modeling we can explore possible types of planet systems, but if we base our instruments on TPF-C and TPF-I solely on these examples, we may miss out on an unexpected color or spectral signature. Recall that the first Mars surface sampler sent back an apparently positive sign of life, but this was a mistake caused by a trace amount of Martian hydrogen peroxide that had not been anticipated. It is possible that we might be quite satisfied with discovering Earth-like planets and life around other stars, however if we do this with blinders we might miss something else. To guard against missing the unexpected, the only solution is to use as broad a spectral range as is feasible, and to use as high a spectral resolving power as possible. (Needless to say the instrument should be optically efficient too, otherwise we cannot utilize the increased resolving power.) Perhaps the best solution is to use a general-purpose spectrograph, as opposed to a potentially more efficient spectral filter bank.

\section{Is our experience with the Earth and the Solar System sufficient preparation?}

This question presumes that we will do all that we can to understand what an Earthtwin, and a Solar-System twin would look like, if placed around a nearby star. To this we must add theoretical modeling, to see what a different set of planets would look like, and evolutionary modeling to see what the Solar-System planets looked like during the past 4.5 billion years, and in the future.

\section{How do we detect these planets and disks?}

After much debate and study, the US has settled on the dual approach of both a visible coronagraph and an infrared interferometer, and this combination has some very strong merits. However we should still remain alert to other methods. We should also remain open to using other observatories to probe planetary systems, some outstanding examples of which are using HST for transits (unfortunately no longer possible) and spectroscopy, Spitzer for infrared transits and dust emission, and ground-based interferometers for zodiacal light measurements. In the future, JWST in space and extreme adaptive optics on the ground are also of interest.

\section{How do we solve the 'physics problem' of detection and characterization?}

The 'physics problem' is the question of how build an optical system that will reduce the star's diffracted and scattered light to a low enough level that we can see the direct light from a planet without significant interference. At present we have a wonderful array of potential techniques to do both of these, as discussed in many papers during 
this conference. It is remarkable that most of these methods cannot be found in optics textbooks, because they have been newly invented specifically for planet-finding. We still need more such inventions. However today we have in hand techniques that will allow us to begin designing TPF-C and TPF-I, and to refine the design as we go along. We need to continue to carry out laboratory demonstrations, and to work on theoretical methods of improving our current designs.

\section{Are precursor missions needed?}

This is a difficult question. The pro argument says that a precursor mission (e.g., a small version of the ultimately-planned TPF-C or TPF-I) is valuable because it provides technological development and experience at a reduced cost, thereby reducing risk in the follow-on full-scale mission. The con side says that there is a political risk in flying a precursor mission because it may turn out that people will mentally convert it to the final mission, and therefore never fund the latter. One way out of this is to do the equivalent of a precursor mission under a different heading, and at a strictly capped cost, so that the small mission cannot easily be viewed as being the same as the final one.

\section{Is international cooperation necessary?}

Cooperation is good in principle (to eliminate duplication, and waste of resources), but can be difficult in practice (culture clashes, trade and information restrictions). My view is that we should work hard to develop international cooperation to help ourselves search for and characterize extrasolar planets, because this is a project for which there is strong interest by the people of all nations, but which will be expensive enough that sharing the cost is a prudent step. We already have in place official agreements to initiate joint work between Europe and the US on the TPF-I/Darwin concept. We should consider expanding this agreement to cover the TPF-C project, and to include other interested nations, such as Japan.

\section{Can we open this new branch of astrophysics, and also provide benefits to other branches?}

Realizing that TPF-C and TPF-I are potentially expensive projects, and that funding is always limited, it would be wise for us to design these missions to serve as broad a segment of the astrophysics community as is possible. Given that these missions have much greater power in areas such as collecting area, angular resolution, wavelength coverage, stability, and other key aspects, it seems logical that more traditional areas of astrophysics could benefit from shared use of mission time. We should actively explore these areas of cooperation, and engage our colleagues in the process from the start.

\section{How do we prepare for the public response to an Earth-like planet? Or to life? Or to no terrestrial planets?}

We all believe that it would be a historically major event to discover a planet that looks like the Earth, and perhaps has life on it. It would be major because people have wondered about such questions throughout the ages. We are highly privileged to live in a time when this question can be answered, and even more privileged to be the scientists who can make these discoveries. Even the statement from us that we have searched high and low for Earth-like planets but found nothing, would be a major discovery. We should 
think about how we want to approach these topics, especially in our dealings with the public, first to be careful to explain that this is good science, and second to not overpromise results. We should also think about what we would say if a reporter calls up and asks what we think about such a discovery. It is possible that the public will see such a discovery as not being important to their daily lives. But I suspect that instead it will be seen as a major turning point, analogous to the time when Copernicus moved the center of the universe from the Earth to the Sun.

\section{An allegorical afterword.}

This year we had the exhilarating experience of being on the verge of a new start for TPF, closely followed by the dispiriting experience of having our funding drastically reduced. This was not the first life-death episode for TPF, nor is it likely to be the last, judging from the history of other missions. The need for governments to spend their resources on other projects will always be with us, just like floods, famine, hurricanes, and wars will always be with us (and in part just because of these things). We must have the confidence of our convictions that the search for extrasolar planets, and their characterization, is a long-term project of major scientific, and societal, importance. Therefore we must weather these changes, and be prepared for the opportunity when better times return.

These thoughts bring to mind the allegory 'Pilgrim's Progress', written in 1678 by John Bunyan in England. Bunyan was thinking of religion in this book, but the images can easily be applied to our labors as well. Specifically, Bunyan envisioned the journey of a person, the eponymous pilgrim, from a land of troubles, down a long road filled with tests of pilgrim's resolution, to the promised land beyond. Our journey from this year to the one in which we have an operating mission, is similar. Pilgrim first passed through the Slough of Despond (that's us today, of course). He then encountered Mr. Worldly Wiseman, the Evangelist, Mr. Obstinate, Mr. Legality, Messrs. Passsion, Patience, Despair, Simple, Sloth, Presumption, Mistrust, and Timorous. Pilgrim climbs the Hill of Difficulty, enters the valley of Humiliation, the Valley of the Shadow of Death, and many more such trials. He finally climbs the Delectable Mountains, and enters the Coelestial City. It was easy for Bunyan's readers to apply the allegory to their lives, and just as easy for us to apply it to our enterprise today. Pilgrim learns the lesson that dedication and steadfastness were the keys to achieving his goal, and so they can be for us.

\section{References}

Bunyan, J. 1678 The Pilgrim's Progress, Barnes and Nobel Classics, New York, 2005 